\title{
Leta Hollingworth, una voz herética en psicología ${ }^{1}$
}

\author{
Carmen García Colmenares \\ Universidad de Valladolid \\ cgcolmen@psi.uva.es
}

\section{Las grietas en la impermeabilidad canónica}

En los últimos años se viene recuperando el papel jugado por las primeras psicólogas, junto con las dificultades que tuvieron que superar debido, principalmente, a barreras de carácter institucional y social (García Dauder, 2005; Scarborough y Furumoto, 1987). Aunque la reconstrucción genealógica de estas pioneras no debe responder solamente a la creación de una historia compensatoria que destaque sus contribuciones desde los cánones académicos tradicionales (Lerner 1992) ni tampoco a rellenar nichos vacíos de la historia de la disciplina (Furumoto, 1992). Algo en si mismo meritorio, pero insuficiente. El interés debe situarse en la elaboración de una historia de las primeras psicólogas "en sus propios términos" (Lerner 1992), destacando los mecanismos de exclusión utilizados (ideológicos, institucionales y personales) que impidieron el acceso a la academia y cómo todo ello afectó a su vida personal y profesional. Así como las estrategias de resistencia que tuvieron que utilizar para subsistir en un contexto tan hostil (García Dauder, 2005; Scarborough y Furumoto, 1987).

La consideración de las mujeres como faltas de capacidad intelectual, justificaría su invisibilidad en la producción científica, que se irá transmitiendo a través del pensamiento misógino y del simbolismo de género (García Colmenares, en prensa; Harding, 1986). Es notoria y reconocida la misoginia de los padres de la psicología como Hall, Boring, o Titchener. Este último como presidente de la Asociación de Psicología Experimental prohibió la admisión de las mujeres en la misma hasta después de su muerte, en 1929 (Furumoto, 1992).

Uno de los mecanismos de reconocimiento para los varones, pero que actuaba de exclusión para las mujeres, era la necesidad de poseer el título de doctor, algo que estaba prohibido a las mujeres en determinadas universidades norteamericanas en los primeros años del siglo XX. Los varones controlaban así la ciencia básica, estando ellas relegadas a la aplicada (Rossiter, 1982).El destino de muchas de ellas será ser profesoras en los colleges femeninos, donde no se contaba con laboratorios tan bien equipados como los de las universidades masculinas, junto con una mayor carga docente. El desprestigio de los colleges lo manifiesta el mismo Williams James en 1898 cuando comentaba que se odiaría a si mismo si estuviese bajo "un régimen de enaguas" (Furumoto, 1987). La vida en estos centros, al contrario que en la competitivas universidades masculinas, hacia que las profesoras

\footnotetext{
1 Antes de comenzar este reflexión sobre el capitulo de Leta Hollingworth me gustaría dar mi agradecimientoa las traductoras,Adriana Velásquez y Maria Ponce de León.
} 
“...no antepusieron sus carreras individuales a su mentalidad comunal como to hicieron muchos profesionales varones; ni se adhirieron acriticamente al racionalismo científico, la especialización, la objetividad de la ciencia social, o la asociación jerárquica en la cual la movilidad vertical tomaba precedencia sobre la hermandad." (Palmieri, 1983: 209-210).

A pesar de los obstáculos, podemos hablar de un número considerables de psicólogas con una interesante obra. Entre ellas podemos citar a Mary Whiton Calkins (1863-1930) y Margaret Washburn (1871-1939), primeras mujeres doctoras en psicología, además de presidentas de la American Psychology Association, junto con Leta Hollingworth, o la combativa Cristine Ladd-Flankin que recibió el titulo oficial de doctora, 44 años después de haberlo obtenido. Son mujeres destacadas en su época y pertenecientes a fuertes colegios invisibles (Tortosa et al., 1987), pero que la falta de transmisión posterior (Birulés, 1997), en citas y referencias ha hecho hayan desaparecido del canon académico, al contrario que sus compañeros masculinos, muchos de ellos peor considerados, que les han sobrevivido en las referencias canónicas textuales y son citados constantemente en los textos y programas académicos. Junto a las trabas institucionales, Scarborough y Furumoto (1987), encuentran dos aspectos temáticos fuente de conflictos: lazos familiares versus carrera profesional y la falta de oportunidades.

La revisión del canon va a permitir rescatar una serie de voces heréticas (Hardy, 1986),así como la reflexión acerca de los mecanismos utilizados para excluir a las y los otros (no varones, no blancos, no heteros). Aunque esta autora nos previne acerca de la tentación de construir una genealogía femenina, ya que se puede producir el efecto contrario y en lugar de debilitar y cuestionar el canon existente lo refuerce, por lo que propone la construcción de un polylogo, que sería

"... el interjuego de muchas voces, una suerte de 'barbarie' creativa que pudiera desbaratar las vías monológicas, colonizantes, céntricas, de la 'civilización'. Tal visión vive, como nos lo ha enseñado Adrianne Rich, en una revisión: una relectura excéntrica, re- descubriendo lo que el manto sacerdotal del canon debería ocultar: la imbricación de toda literatura con la dinámica del poder en la cultura." (Hardy, 1986, 298)

La crítica al canon debe ir, por tanto, más allá de la mera la oposición entre dentro- fuera, superando esa dicotomía, desplazando el análisis y facilitando "una visión desde otro lugar", tal como señala Teresa de Lauretis,

"... este 'otro lugar' no es un pasado mítico o distante, o una utópica historia futura, es el otro lugar del discurso aquí y ahora, el punto ciego, el fuera de campo de sus representaciones. Me lo imagino como espacios a los márgenes de los discursos dominantes, espacios sociales enclavados en los intersticios de las instituciones, en las fisuras y en las grietas de los aparatos del poder saber. En estos espacios pueden ponerse los términos de una construcción distinta del género, términos que tienen efecto y se afianzan en el nivel de la subjetividad y de la autorrepresentación: esto es, las prácticas micropolíticas de la vida diaria y de las resistencias cotidianas, de las que derivan tanto la capacidad de obrar como las fuentes del poder y las inversiones que otorga el poder; y también en la producción cultural de las mujeres, feministas, que traduce el movimiento dentro y fuera de la ideología en un continuo 
atravesar los confines (y los límites de la/s diferencia/s sexual/es)." (Lauretis, 1987/2000: 62).

Leta Anna Stetter nace el 25 de mayo de 1886, en el estado de Nebraska en cuya Universidad estudia lengua y literatura. Obtenida la diplomatura a los 19 años enseña en el instituto de Nebraska durante dos años, para mas tarde trasladarse a Nueva York, donde su futuro marido es ayudante de James Mckeen Cattell en la Universidad de Columbia. Y aunque Leta tenía intención de dedicarse a la enseñanza, el estar casada fue un impedimento para ello. Una de sus mejores biógrafas, Linda Silverman nos recuerda:

"Como era una escritora con talento, intentó escribir cuentos, pero fueron rechazados por los editores. Busco una beca para estudiar en la universidad de Columbia, pero sus planes se vieron frustrados. Desconcertada, intentó adaptarse a las tareas domesticas, pasando los días cosiendo, remendando, cocinando, lavando planchando y limpiando, hasta que la frustración de no poder hacer otra cosa la abrumó. Una mañana del otoño de 1910 , se puso a despotricar con Harry (su marido) sobre la injusticia de su situación, se echo a llorar y después decidió hacer algo para cambiarla-no solo para si misma, sino para todas las mujeres que estuvieran presas en su mismo apuro. Fue a la biblioteca de la universidad y se puso a devorar todo lo que cayó en sus manos sobre por qué se les negaba a las mujeres el acceso al trabajo ya las oportunidades educativas. Pertrechada con las teorías y la 'investigación' de su tiempo, comenzó a desacreditar a todas las afirmaciones sobre la inferioridad natural de las mujeres." (Silverman, 1999: 40 [la cursiva es nuestra]).

Al año siguiente comienza los estudios de postgrado en la Universidad de Columbia y obtiene el Master en Educación en 1913 con la tesis sobre la Periodicidad Funcional donde atacaba la idea de que las mujeres durante la menstruación eran menos productivas que los varones, poniendo en entredicho la variable biológica como coartada para negarles trabajo y pagarles menos. Al poco tiempo va al Clearing House for Defectives Mental de Nueva York, donde continua sus investigaciones sobre las mujeres; en 1914 consigue el puesto de psicóloga en la ciudad de Nueva York y realiza sus primeras publicaciones. En 1916 obtiene su doctorado en educación y comienza a trabajar como profesora de psicología de la educación en la Universidad de Columbia.

Sus intereses científicos son muy amplios, destacando además de su militante feminismo, en investigaciones sobre superdotación y en el tema que nos ocupa, las supuestas diferencias aptitudinales y vocacionales ente varones y mujeres. Para un estudio más exhaustivo de la autora pueden consultarse una serie de biografías sobre su vida y obra (Klein, 2000; Hollingwoth, 1943; Rossiter, 1992). En castellano puede consultarse las referencias ya citadas de Silverman y Silvia García Dauder.

\section{Las primeras psicólogas españolas, otro secreto bien guardado}

Antes de adentrarnos en el análisis de la obra de Hollingworth, me gustaría apuntar, al menos brevemente, la situación en nuestro país de las primeras mujeres psicólogas, puesto que al igual que las estadounidensez, también han sido un secreto muy bien guardado (Scarboroug y Furumoto, 
1987). En estos momentos y gracias al disfrute de un año sabático ${ }^{2}$ estoy realizando una investigación sobre la reconstrucción biográfica de las psicólogas fundacionales españolas, que me esta permitiendo conocer su trayectoria vital y profesional.

Las barreras institucionales les impedirán el acceso a la universidad hasta 1910, teniendo que solicitar un permiso especial, como ocurrió con Concepción Arenal que tuvo que asistir a la universidad disfrazada de varón. Esta autora, aunque abogada de profesión, fue muy crítica con las teorías de la época acerca de la inferioridad intelectual de las mujeres. En su obra La mujer del porvenir (1868/1993) resalta los planteamientos misóginos de autores como Gall, planteamientos que no eran percibidos por otros ilustres colegas de la época. Superada la prohibición expresa, las mujeres españolas tendrán que superar otros impedimentos basados en los prejuicios religiosos y sociales de la época.

Para salvar las prohibiciones de acceso a la universidad, las primeras psicólogas españolas estudiarán magisterio en las diferentes Escuelas Normales del país. Obtenido el título de maestra superior, una forma de promoción profesional y ampliación de estudios era el acceso a la Escuela Superior del Magisterio en Madrid, creada en 1909 (por Molero y Pozo) que permitía el perfeccionamiento pedagógico y las posibilidades de ser profesoras en Escuelas Normales. Es el caso de Dolores González Blanco, Regina Lago o Maria Luisa Navarro. Las estudiantes que procedían de provincias alejadas de Madrid, tenían que quedarse en régimen de pensión. El lugar elegido será la Residencia de Señoritas, creada en (1915) y dirigida por Maria de Maeztu. En ella contaban con un entorno intelectual con posibilidades de acceso a archivos, bibliotecas, laboratorios, en semejanza a los colleges de EE.UU. Algunas de las primeras psicólogas serán alumnas de dicha residencia, como Lago que en el año 36 sustituirá a Maria de Maeztu como directora de la misma y que se formó en psicología experimental y educación en Suiza junto con Claparede y Piaget.

De las cinco psicólogas españolas que estamos estudiando en estos momentos, tres fueron profesoras de Escuelas Normales, González Blanco, Lago y Maria Luisa Navarro. Concepción Sáiz de Otero fue profesora de las tres en la Escuela Superior de Estudios del Magisterio de Madrid y Mercedes Rodrigo, aunque hizo los estudios del magisterio se especializó en la orientación profesional.

Una forma de resistencia ante las limitaciones que suponía la docencia en las Escuelas Normales fue la posibilidad de ser pensionadas (becadas) por la Junta de Ampliación de Estudios (JAE), creada en 1907 y que permitía formarse fuera de España, principalmente en Francia, Alemania y Suiza, aunque también algunas fueron a Inglaterra y Estados Unidos. Para obtener estas ayudas se necesitaba tener un buen currículo académico, publicaciones y hablar varios idiomas, siendo las profesoras de Escuelas Normales las únicas mujeres profesionales que superaron a los varones en cuanto al número en la concesión de dichas pensiones (Marin, 1990). Sáiz de Otero fue pensionada una sola vez para viajar a Londres en 1908; Rodrigo lo fue cuatro veces en diferentes lugares como Francia, Bélgica, Suiza y Alemania, la primera vez en 1920; González, Navarro y Lago, dos veces cada una en países como Francia Bélgica, Suiza y Alemania. Su formación la realizaron en universidades como la

\footnotetext{
${ }^{2}$ A través delproyecto titulado Autoridad femenina y genealogías en la psicología española y de América Latina, he podido conocerla vida y obra de un interesante número de pioneras rastreando archivos tanto de diferentes comunidades del estado español como de países latinoamericanos (Argentina, México y Chile), donde alguna de ellas tuvieron que exiliarse después de la guerra civil.
} 
Sorbona, el Instituto J.J. Rousseau de Ginebra dirigido por Claparede, y en los afamados laboratorios de Psicología en Alemania.

Todas ellas serán coetáneas de Hollingworth y al igual que ella, tuvieron un destacado protagonismo en la lucha por la emancipación de las mujeres, siendo la mayor de todas ella, Sáiz de Otero, considerada una de las pioneras del feminismo en nuestro país (Scalon,1986). Dejaran una importante obra escrita que estamos intentando rescatar a pesar de su dispersión, descatalogación y falta de transmisión.

Todas ellas, excepto Saiz de Otero, fueron perseguidas durante la guerra civil por lo que se tuvieron que exiliarse; Rodrigo en Colombia, Navarro en Argentina y Lago en México. González Blanco, permaneció en España y fue depurada y destituida de su cargo como profesora de la Escuela Normal. Pero esto es otra historia que contaremos en otro momento. Prosigamos con el artículo de Hollingworth.

\section{Las aptitudes vocacionales de las mujeres, revisión de un viejo problema}

El artículo que vamos a analizar fue publicado dentro de la obra Vocacional Psychology, dirigida por su marido Harry Hollingworth. En el mismo encontramos una serie de temas muy queridos para nuestra autora como son la polémica acerca diferencias sexuales en inteligencia y vocación profesional, la falta de control de las mujeres durante la menstruación, la variabilidad intelectual de la varones frente a la falta de la misma en las mujeres, el instinto maternal y la división sexual del trabajo. La claridad expositiva de la autora, que de manera sistemática va desmontando las diferentes cuestiones que se plantean, me permite tomar la licencia de centrarme solamente en algunos aspectos en los que Leta tuvo un importante papel como investigadora. Y más que realizar un análisis exhaustivo del presente articulo, que tiene un indudable interés divulgativo, me gustaría presentar algunas claves que puedan ayudar a contextualizar " en sus propios términos" este trabajo y resaltar la vigencia de los temas no solamente para la disciplina psicológica sino también para el actual debate dentro de las diferentes corrientes del feminismo. Así pues, comentaré con más de detalle la cuestión segunda y cuarta, referidas respectivamente a la variabilidad mental y al instinto maternal.

Tal como ella misma nos cuenta

“...esta segunda pregunta sería de gran importancia para la orientación vocacional. Por ejemplo, si los datos experimentales demostraran que las mujeres son más bien parecidas entre sí, por naturaleza, mientras que los hombres se diferencian ampliamente unos de otros, tendríamos una base científica para concluir que la justicia y la economía social son correctas conforme a la política actual de orientar a todas las muchachas hacia una única ocupación, mientras que a los muchachos se les incentiva a acceder al mayor número posible de profesiones" (Hollingwoth, 1914: 4-5).

Después de insistir en la inconsistencia de la bibliografía de opinión sobre el tema, señala una serie de investigaciones que evidencian la no existencia de diferencias en la variabilidad mental entre los sexos. 
En otro texto, del que ella es también autora, Variability as related to sex differences in achievement. A critique, publicado en el American Journal of Sociology en 1914, ahonda no solamente en las cuestiones estadísticas, sino también en la base social de la variabilidad mental masculina que los padres de la psicología justificaban por la escasa presencia de jóvenes mujeres en centros asistenciales al contrario de lo que ocurría con los varones jóvenes discapacitados.

"El chico que no puede competir mentalmente es investigado, se convierte en la edad más temprana en objeto de preocupación para sus parientes, es ingresado en Clearing House, y dirigido hacia una institución. La chica que no puede competir mentalmente no es a menudo reconocida como definitivamente discapacitada, como quiera que no es antinatural para ella quedarse en el aislamiento de la casa donde puede "cuidar de" los niños pequeños, pelar patatas, etc. Si es físicamente pasable, como a menudo es el caso, se puede casar y asegurarse así el apoyo económico, o se puede convertir en prostituta para conseguir soporte económico, para lo cual su mentalidad débil no es una barrera." (Hollingwoth, 1914: 516-517).

La variabilidad intelectual de ellos, les permitiría situarse tanto en el extremo superior como inferior de la campana de Gauss, mientras que la falta de variabilidad de las mujeres, las agrupaba las zonas centrales de la misma. A través de artificios estadísticos como la campana de Gauss y los tests de inteligencia, la psicología oficial justificaba las diferencias sexuales en la excepcionalidad intelectual de los varones que permitía su participación en el espacio público, olvidando la incidencia de una serie de variables como con la etnia, la clase social o el sexo/género. La homogenización de las mujeres, las excluía de la imbecilidad profunda, pero a costa de no reconocerles la genialidad, englobándolas en las medianías mentales (García Colmenares, en prensa). En palabras de Celia Amorós (1985) los varones ocuparían el espacio de los iguales frente a las mujeres que permanecerían en el de las idénticas.

"Todo derecho a la diferencia presupone, obviamente, la igualdad; de otro modo mi diferencia no se vería reconocida, es decir, ponderada como digna del mismo respeto que la del otro... En contraposición, pues, con los enunciados de identidad, aquellos en los que se expresa igualdad implican la discernibilidad de los términos que homologan....Querríamos poner de manifiesto como la igualdad se ha venido solapando históricamente como la fraternidad entendida como fratría de los varones, mientras que la identidad tendría en el genérico femenino su supremo análogante." (Amorós, 1985: 31-32).

La identidad supone volverse indiscernible como sujeto al tener como marcas de referencia el carácter grupal y no individual que, en el caso de las mujeres, nos llevaría a la esencialización a través del concepto Mujer. Tal como señala Carole Patterman (1988), la idea de sujeto e igualdad es masculina, lo que lleva a la consecución de derechos civiles como el voto en la revolución francesa, mientras que no ocurre lo mismo en las mujeres. La negación del reconocimiento de la individualidad de las mujeres conlleva la negación del poder a las mismas (Amorós 1985; Patterman, 1988).

Hollingworth en sus investigaciones sobre superdotación encontrará mujeres con altas capacidades en igual número que varones pero distinguirá claramente entre alta capacidad y eminencia, enfrentándose a autores como Galton que consideraba que la eminencia era signo de alta capacidad.

"Parece indudable que un gran número de mujeres de talento intelectual, enfrentadas a la elección entre 'carrera' y 'felicidad doméstica', han elegido, tanto 
consciente como inconscientemente, la última. Y debemos recordar que la misma opción de elegir ha existido sólo recientemente, que durante casi todo el curso de la historia, las mujeres fueron predestinadas al trabajo doméstico. No se sabe y no se puede saber qué grado y en qué cantidad el potencial de liderazgo ha sido desviado a los canales de absorción de energía dónde la eminencia es imposible. Las tareas domésticas y el cuidado de los hijos, aunque mucho se encomendó a las mujeres como ámbitos apropiados para la explotación de sus talentos, son, desgraciadamente por su gama, no los ámbitos en los que se puede encontrar la eminencia". (Hollingworth, 1914: 527).

Otro tema de gran vigencia en la actualidad es el relacionado con el instinto maternal que nuestra autora, haciendo una brillante analogía, le compara con el trabajo de los soldados

"El alumbramiento y crianza de los niños se parece, en muchos aspectos, al trabajo de los soldados: es necesario para la existencia de la nación, implica grandes sacrificios personales, conlleva sufrimiento, peligro y, en cierto porcentaje de casos, incluso la muerte. Por lo tanto, como en el caso de los soldados, se deben realizar todos los esfuerzos necesarios para establecer como norma el extremo de la curva de distribución donde se encuentra un ferviente interés por el patriotismo, en este caso, y por la maternidad en el otro. A falta de datos científicos debemos evitar, por consiguiente, aceptar como hecho establecido de la naturaleza humana una doctrina que posiblemente encontraremos funcionando como medio de control social." (Hollingworth, 1914: 10).

En 1916 Hollingworth en el articulo Social devices for impelling women to bear and rear children cuestionará la mística del instinto maternal apelando de nuevo a la bibliografía de los hechos al plantear que denominado instinto se mantiene gracias a una serie de dispositivos institucionales y de control social que son utilizados por los guardianes sociales, principalmente el personal que ejerce la medicina y la psicología, a través de tres mecanismos como: la construcción de silencios sobre los aspectos dolorosos o menos gratos de la gestación, el parto y el cuidado de las criaturas; la exaltación de la maternidad; y los miedos, amenazas sobre las consecuencias de no ser madre. Es el miedo recurrente a ser una mujer superflua o redundante (Faderman, 1981).

Esperamos, con estas líneas, haber suscitado el interés en las y los lectores hacia la vida y la obra de Hollingowrth y con ello contribuir, en alguna medida, a un mejor conocimiento de sus aportaciones. Al igual que ella acabamos nuestro comentario con sus mismas palabras, a pesar del largo tiempo trascurrido:

"Por lo que hasta estos momentos se sabe, las mujeres son intelectualmente tan competentes como los hombres para seguir todas y cada una de las vocaciones humanas".

\section{Referencias}

Arenal, Concepción. (1868/1993). La mujer del porvenir. Madrid: Castalia. Instituto de la Mujer. 
Birulés, Fina. (1997). Indicios y fragmentos: historia de la filosofía de las mujeres. En Rose María Rodríguez Magda (ed.). Mujeres en la historia del pensamiento (pp. 17-31). Barcelona: Anthropos.

Faderman, Lillian. (1981). Surpassing the love of men: Romantic friendship and love between women from de renaissance to present. Nueva York: Morrow.

Furumoto, Laurel. (1987). On the margins: Women and professonalizacion of Psychology in the United States. 1890- 1940. En Michell G. Ash y Williams R. Woodward (eds). Psychologie in twentieth century and society (pp. 93-113). Cambridge, Nueva York: Cambridge University Press.

Furumoto, Laurel. (1992). The new History of Psychology. En Janis S. Bohan (ed). Re-placing women in Psychology. Readings toward a more inclusive History (pp. 11-30). lowa: Kendall/Hunt Publishing Company.

García Dauder, Silvia. (2005). Psicología y Feminismo. Madrid: Narcea.

García Colmenares, Carmen. (en prensa). Autoridad femenina y mecanismos de exclusión. Reflexiones desde la psicología. En Alicia Puleo (coord.) Filosofía, Igualdad y Género. XII Jornadas. Valladolid: Departamento de Filosofía. Universidad de Valladolid.

Hardy, Susan. (1986). Women and the Question of Canonicity. College English, 48, 3, 288-299.

Harding, Sandra. (1993). The science question in feminism. Ithaca, N. York: Cornell University Press.

Hollingworth, Harry. (1943). Leta Setter Hollingworth: A biogrphy. Lincoln: University of Nebraska Press.

Hollingworth, Letta. (1914). Variability as related to sex differences in achievement. A critique. The American Journal of Sociology,19, 510-530.

Hollingworth, Letta. (1916). Social devices for impelling women to bear and rear children. The American Journal of Sociology, 22, 19-29.

Klein, Ann G. (2002). A forgotten voice. A biography of Leta Setter Hollingworth. Scottsdale: Great Potencial Press.

Lerner, Gerda. (1992). Placing women in History: definitions and challenges. En Janis S.Bohan (ed). Re-placing women in Psychology. Readings toward a more inclusive History (pp. 31-43). lowa: Kendall Hunt Publishing Company.

Lauretis Teresa. (1987/2000). La tecnología del género. En Teresa Lauretis. Diferencias (pp. 33-64). Madrid: Horas y Horas.

Marin, Teresa. (1990). La renovación pedagógica en España (1907-1936). Los pensionados en pedagogía por la Junta de ampliación de estudios. Madrid: Consejo Superior de Investigaciones Científicas.

Molero, Antonioy Pozo, Ma del Mar. (1989). La Escuela de Estudios Superiores del Magisterio (19091932). Alcalá de Henares: publicaciones Universidad de Alcalá. 
Palmieri, Patricia A. (1983). Here was fellowship: a social portrait of academic women at Wellesley College, 1985-1920. History of education Quarterly, 23, 195-214.

Patterman, Carole. (1988). The sexual contract. California: Stanford University Press.

Rossiter, Margaret. (1982). Women Scientists in America. Struggles and Strategies to 1940 Baltimore. Londres: The Johns Hopkins University Press.

Tortosa, Francisco; Carpintero, Helio y Peiró, Jose María. (1987). La psicología americana a través de la American Journal of Psychology. Revista Historia de la Psicología, 8, 1-2, 5-37.

Scarborough, Elisabeth y Furumoto, Laurel. (1987). Untold lives: The first generation of American women psychologist. Nueva York: Columbia University Press.

Silverman, Linda. (1999). Todo empezó con Letta Hollingworth: historia de la superdotación en las mujeres. En Julie Ellis y John Willinsky (ed.). Niñas, mujeres y superdotación. (pp. 37-50). Madrid: Narcea.

Scalon, Geraldine. (1986). La polémica feminista en la España contemporánea (1868-1974). Madrid: Akal.

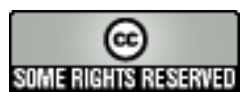

Este texto está protegido por una licencia Creative Commons.

Usted es libre de copiar, distribuir y comunicar públicamente la obra bajo las siguientes condiciones:

Reconocimiento: Debe reconocer y citar al autor original.

No comercial. No puede utilizar esta obra para fines comerciales.

Sin obras derivadas. No se puede alterar, transformar, o generar una obra derivada a partir de esta obra.

\section{$\underline{\text { Resumen de licencia }}$}

$\underline{\text { Texto completo de la licencia }}$ 